\title{
New Australian NePenthes taxa PUBLISHED SINCE ALLEN LOWRIE's MAGNUM OpUS
}

RICHARD NunN • Sydney•Australia • richardjnunn1@gmail.com

Nepenthes L. is a genus comprising at least 160 accepted species chiefly distributed in Southeast Asia, with centres of diversity in Borneo, Sumatra, and the Philippines, with disjunctions in Madagascar, Sri Lanka, India, Seychelles, New Caledonia, and Australia (Clarke et al. 2018).

The number of Nepenthes species recorded in Australia has changed greatly since the days of Bailey $(1881,1897,1898,1899,1905)$ and von Mueller (1866) who reported 11 species, and Danser (1928) who subsequently reduced them to synonyms of $N$. mirabilis (Lour.) Druce. Treatments of the genus by Stanley (1982), Jebb and Cheek (1997), and Cheek and Jebb (2001) followed Danser. Clarke and Kruger (2005), after viewing Bailey's original type material held at Brisbane Herbarium (BRI) and detailed field observations, re-instated one of Bailey's collections, N. rowaniae F.M.Bailey, as a distinct species. Whilst completing these field studies Clarke and Kruger encountered a Nepenthes taxon that was not readily identifiable and didn't match any herbarium specimens, which they concluded was a new species and described N. tenax C.Clarke \& R.Kruger in 2006. Lowrie (2014) supported three species, N. mirabilis, N. rowaniae, and N. tenax in Magnum Opus and this treatment remained the accepted protocol until 2016, when a fourth Australian species was described, Nepenthes parvula G.W.Wilson \& S.Venter.

\section{Nepenthes parvula G.W.Wilson \& S.Venter 2016}

Clarke \& Kruger (2006) found a single population of a "small form" of Nepenthes tenax growing in a permanently-inundated site in a swamp on the flood plain of the Jardine River and they observed that this form "bears the smallest functional aerial pitchers of any $\mathrm{Ne}$ penthes" with pitchers that are "at most 50 $\mathrm{mm}$ high". Other personal accounts of this diminutive taxon have been recorded after field studies of the Jardine River area. The authors of this new taxon (Wilson \& Ventor 2016) conducted several field surveys in northern Queensland during the Dry Season (May to November) from 2010-2015 to northern Cape York. The population of the proposed new taxon appeared clearly distinct from $N$. tenax on basis of morphological and ecological characteristics and lead to its formal description in 2016.

Nepenthes parvula is an erect subshrub 0.35(-0.50) $\mathrm{m}$ tall (Fig. 1). The epithet parvula refers to the small size of mature plants. Nepenthes parvula occurs in Queensland, Cape York only growing on freshwater swamps in the lower Jardine River catchment

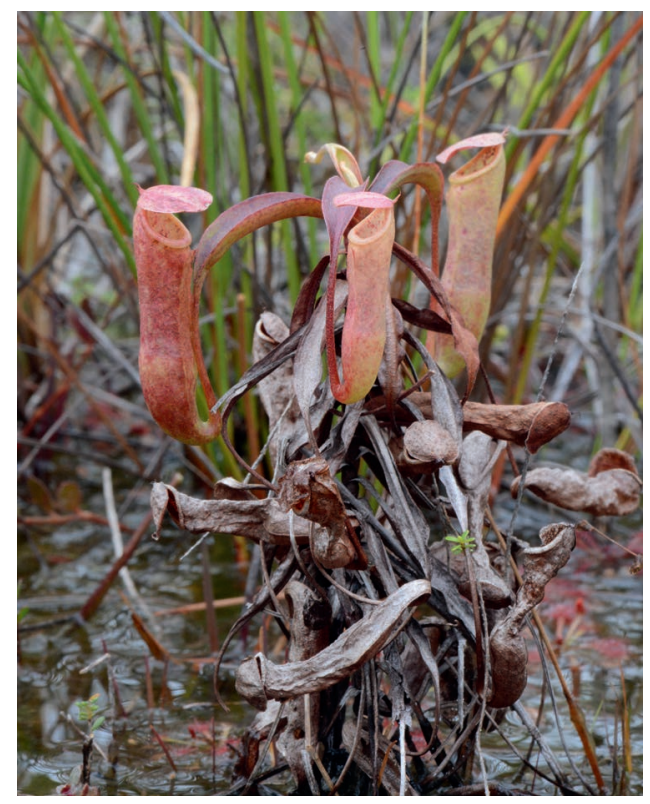

Figure 1: Nepenthes parvula growing in Sanamere Lagoon, Cape York, Queensland, Australia. Photo by Richard Nunn. 


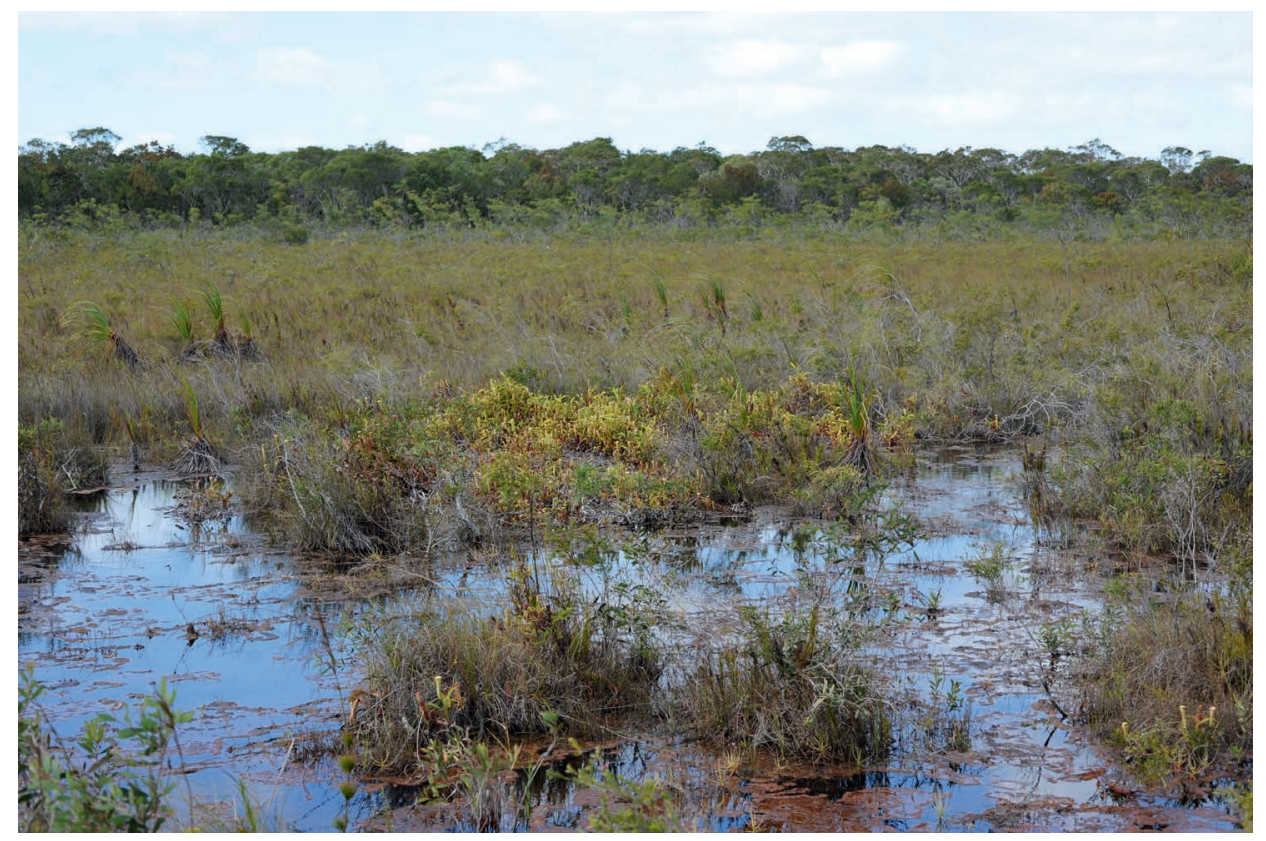

Figure 2: Typical habitat for Nepenthes parvula, Sanamere Lagoon, Cape York, Queensland, Australia. Photo by Richard Nunn.

(Fig. 2). This ecosystem does not burn in the wildfires that irregularly but not infrequently sweep across the landscape and as result $N$. parvula is characterised by a retained "skirt" of lower pitchers. Nepenthes parvula is similar to $N$. tenax from which it differs in having small aerial pitchers (35-60 $\times 10-15 \mathrm{~mm}$ ), red colour of the upper surface of the lid on aerial pitchers, more dense nectar glands (250-300 per $\mathrm{cm}^{2}$ vs. $100-150$ per $\mathrm{cm}^{2}$ ) on the abaxial surface of the pitcher lid, smaller male flower, much shorter mature fruit, and restriction to an ever-wet environment.

Robinson (2020) in Flora of Australia suggests the principally size-based distinction is poorly supported genus-wide given the inherent variability of even single species populations studied across the range of the genus, and in-situ comparisons of $N$. tenax and $N$. parvula found that it was sometimes challenging to distinguish between them, with various intergrades noted (A. Robinson, pers. obs).

However, Wilson and Venter (2016) indicate that the ecology of this taxon is distinct, and that plants of $N$. tenax and $N$. parvula grown under identical conditions respond differently, particularly in terms of their temperature tolerances (Wilson \& Venter 2016). It is worth noting that the Sulawesian $N$. minima is likewise separated from $N$. maxima based primarily on its diminutive dimensions, appearing otherwise much like its larger counterpart, but genetic analyses seem to support this separation (Nauheimer et al. 2019) and similar analyses of the Australian Nepenthes taxa may provide valuable insight into the quality of their separation.

\section{References}

Bailey, F.M. 1881. On a new species of Nepenthes. Proceedings of the Linnean Society of New

South Wales 5: 185-187. http://dx.doi.org/10.5962/bhl.part.15875.

Bailey, F.M. 1897. Contributions to the Flora of Queensland. Queensland Agricultural Journal 1:

228-235. 
Bailey, F.M. 1898. Contributions to the Flora of Queensland. Queensland Agricultural Journal 3: 353-356.

Bailey, F.M. 1900. Contributions to the Flora of Queensland. Queensland Agricultural Journal 7: 441.

Bailey, F.M. 1905. Contributions to the Flora of Queensland. Queensland Agricultural Journal 16: 189-193.

Cheek, M., and Jebb, M. 2001. Nepenthaceae Dumont. In: Nooteboom, H.P. (ed.), Flora Malesiana, vol. 15. Nationaal Herbarium Nederland, Leiden, pp. 1-157.

Clarke, C.M., and Kruger, R. 2005. Nepenthes rowanae (Nepenthaceae), a remarkable species from Cape York, Australia. Carnivorous Plant Newsletter 34(2): 36-41.

Clarke, C.M., and Kruger, R. 2006. Nepenthes tenax C.Clarke \& R.Kruger (Nepenthaceae), a new species from Cape York Peninsula, Queensland. Austrobaileya 7 (2): 319-324.

Clarke, C.M., Schlauer, J., Moran, J.A., and Robinson, A.S. 2018. Systematics and evolution of Nepenthes. In: Ellison, A.M. \& Adamec, L. (eds.), Carnivorous Plants: physiology, ecology, and evolution. Oxford University Press, Oxford, pp. 58-69. https://doi.org/10.1093/ oso/9780198779841.003.0005

Danser, B.H. 1928. The Nepenthaceae of the Netherland's Indies. Bulletin du Jardin Botanique de Buitenzorg III 9(3-4): 249-438.

Jebb, M.H.P., and Cheek, M. 1997. A skeletal revision on Nepenthes. Blumea 42: 1-106.

Mueller, F. 1866. Nepenthes, Fragmenta Phytograpiae Australiae 5: 154.

Nauheimer, L., Cui, L., Clarke, C., Crayn, D.M., Bourke, G., and Nargar, K. 2019. Genome skimming provides well resolved plastid and nuclear phylogenies, showing patterns of deep reticulate evolution in the tropical carnivorous plant genus Nepenthes (Caryophyllales). Australian Systematic Botany 32(3): 243-254.

Robinson, A.S. 2020. Nepenthes parvula. In: Kodela, P.G. (ed.), Flora of Australia. Australian Biological Resources Study, Department of Agriculture, Water and the Environment: Canberra. https://profiles.ala.org.au/opus/foa/profile/Nepenthes\%20parvula [Accessed: 22 October 2021].

Stanley, T.D. 1982. Nepenthaceae Dumont. In: George, A.S. (ed.), Flora of Australia, vol. 8. Australian Biological Resources Study, Canberra, pp. 7-8.

Wilson, G.W., and Venter, S. 2016. Nepenthes parvula (Nepenthaceae), a new species from Cape York, Queensland, Australia. Phytotaxa 277 (2): 199-204. http://dx.doi.org/10.11646/phytotaxa. 277.2.7 